\title{
The role of perceptually represented structure in analogical problem solving
}

\author{
RICHARD CATRAMBONE, DAVID L. CRAIG, and NANCY J. NERSESSIAN \\ Georgia Institute of Technology, Atlanta, Georgia
}

\begin{abstract}
Current models of analogical reasoning assume that representations of source examples and target problems occur in an amodal format - that is, a representation whose construction and processing are independent of activity in the perceptual and motor cortices of the brain. We examined the possible use of kinesthetic information-perceptual structures associated with the sensation of space and forcein the representation of source examples and target problems. Participants who recreated a source story while acting out the key elements were more likely to access the story when later working on the target problem than were participants who only verbally recreated the story or who verbally recreated it as well as sketched it. We argue that enactment made kinesthetic and spatial features more salient in participants' source story representations and that this aided performance. These results suggest that current models of analogical reasoning might be improved by including perceptual information as part of their representational schemes.
\end{abstract}

People sometimes solve otherwise intractable problems by forming and exploiting analogies with related source cases. Understanding how source cases and target problems are represented can potentially shed light on how sources are retrieved and how, once retrieved, they are used to make productive inferences. Most research on analogical problem solving, however, has assumed that source and target representations have exclusively amodal, or language-like, forms (e.g., Gentner, Rattermann, \& Forbus, 1993; Gick \& Holyoak, 1980; Keane, Ledgeway, \& Duff, 1994; Wharton et al., 1994). This assumption has not been directly tested, and we argue that it is likely incorrect.

A possible alternative to the amodal assumption is the idea that representations of sources and targets can, in some cases, include perceptually coded information that directly enters into the formation and application of analogies. This possibility is consistent with recent explanations of how concepts could be represented in the perceptual and motor cortices (Barsalou, 1999; Glenberg, 1997), with evidence that many conceptual processes are tied to some form of perceptual simulation (Fincher-Kiefer, 2001; Pecher, Zeelenberg, \& Barsalou, 2003; Solomon \& Barsalou, 2001; Stanfield \& Zwaan, 2001), and with arguments linking abstract reasoning to the use of kinesthetic schemas (Johnson, 1987; Lakoff, 1987; Mandler, 1992; Richardson, Spivey, Edelman, \& Naples, 2001; see also

This research was supported in part by Air Force Office of Scientific Research Grant F49620-98-1-0362 to R.C. and NSF Grant SBR9810913 to N.J.N. Correspondence concerning this article should be addressed to R. Catrambone, School of Psychology, Georgia Institute of Technology, Atlanta, GA 30332-0170 (e-mail: rc7@prism.gatech.edu).

Note-This article was accepted by the previous editorial team, when Colin M. MacLeod was Editor.
Anderson, 1984). Wolff and Song (2003) found evidence that people's understanding of causal verbs is better captured by a model that includes force (the force dynamic model) than by models that are based more on conjunction or covariation.

In this article, our primary focus is on testing whether a particular type of perceptual information, kinesthetic information, can play a role in analogical reasoning. We test the claim that manipulating the likelihood of relevant kinesthetic information being part of source representations while holding other factors constant - can influence the access and application of the source when a person is trying to solve an analogous problem.

\section{Analogical Problem Solving}

Analogical problem solving is the activity of solving new problems, referred to here as targets, by reference to related source situations. The activity can be functionally divided into three processes: the retrieval of a source representation; the mapping of a source representation onto a target representation (identifying exactly which aspects of the two are comparable and which are irrelevant); and the transfer of some aspect of a source, by inference, onto a target. Similarities between a source and a target representation presumably facilitate retrieval and guide the mapping and transfer processes. When representations are viewed as being strictly amodal, retrieval and mapping should be more efficient with respect to goal attainment if the processes are guided more by structural relations in the presentations than by superficial features. This hypothesis is supported by findings that performance on analogical inference and problem solving is enhanced most easily by making structural similarities more explicit or by having people carry out tasks designed to highlight conceptual structure in sources (Gentner et al., 1993; Holyoak \& Koh, 1987). 
Similarly, we argue that the presence of relevant kinesthetic information in a representation can lead to superior performance in analogical problem solving because this information can aid access of relevant prior knowledge.

\section{Perceptual Information and Analogical Reasoning}

Suppose someone reads a story in which a force is imparted on an object from one direction and then from another direction and another and so forth. Perceptual information could be incorporated in a representation of these actions in at least two distinct ways. First, as the story is read, distinct kinesthetic representations of linear force - linear force schemas - might be activated in working memory to code the force relations as they come up. A collection of such schemas would then become part of the way the story is remembered. Alternatively, an altogether different representation of force might be activated to represent the same events. Reference to multiple convergent forces might activate a single perceptual representation of converging force akin to squeezing, rather than activating multiple linear force schemas. Insofar as a single convergent force representation is not the same as the representation of a collection of individual linear forces, this representational difference could, in turn, affect subsequent inferences and problem solving.

To examine how such differences might enter into analogies, consider the analogy between the General story (Gick \& Holyoak, 1980, 1983; see Appendix A) and Duncker's (1945) radiation problem (see Appendix B). The General story tells of a rebel leader planning to attack a fortress with his army. The leader knows he will need the entire army to capture the fortress but learns that the roads leading to the fortress are full of mines that will go off if his entire army marches en masse. To overcome this problem, he splits his men into small groups and sends them down different roads in a coordinated fashion, so that they meet safely at the fortress in full strength. The radiation problem, on the other hand, involves trying to destroy a patient's tumor with a beam of radiation, the explicit constraint being that a beam strong enough to destroy the tumor will destroy any healthy tissue it passes through as well, killing the patient in the process. The analogy lies with the fact that the patient's tumor can also be treated by dividing concentrated resources where they are harmful and reuniting them where they are helpful, or more specifically by using multiple weak beams of radiation centered on the same point.

The radiation problem consists of a set of requirements and constraints, central among which are the constraints that radiation be weak or diffuse around the tumor and strong or intense on it. These constraints, coincidentally, match attributes that can be inferred from the convergent force schema - namely, that the intensity is higher at the center than at the periphery; hence, that schema, if somehow incorporated in a reader's representation of the General story, should help trigger retrieval of the story when one reads the radiation problem. Conversely, if only multiple linear force schemas are part of the representa- tion of the General story, or if no perceptually represented information is part of the representation, the weak-atthe-periphery and strong-at-the-center attributes are relatively unlikely to be present, and this advantage is lost. Of course, there is no reason to expect that a representation of the General story, if read in a conventional context, would include a convergent force schema, insofar as there are no references, explicit or metaphoric, to force. Changing the perceptual context of the story or changing the perceptual context in which it is read, however, could bring the convergent force schema into play.

Pedone, Hummel, and Holyoak (2001) have provided some support for the idea that perceptual information can facilitate solving the radiation problem analogically. Rather than have participants read source stories prior to working on the problem, Pedone et al. showed them source diagrams designed to represent the standard abstract solution principle. Consistent with an earlier finding by Gick and Holyoak (1983), Pedone et al. found that participants who viewed static diagrams showing several arrows converging on a focal point did no better on the target problem than did participants who viewed no diagram. Static diagrams might not have helped because they conveyed a spatial schema without explicitly eliciting a sense of force. Without the force component, the diagrams could not readily convey the fact that intensity is low at the periphery and high at the point of focus, which, as we have argued above, is the central constraint of the radiation problem. Pedone et al. next tried using as a source an animated version of the converging arrow diagram, showing the arrows moving toward the center via blinking dashed lines. An animated diagram was predicted to be particularly effective at eliciting the notion of convergent forces because animated arrows appeared to collide at the center. Consistent with this analysis, people who viewed the animated diagram performed dramatically better on the radiation problem than did those who viewed a static diagram.

The manipulations used by Pedone et al. (2001) were designed to increase the likelihood of participants encoding the source analogue as lines of convergence, yet their account of the representations used in analogical inference is essentially amodal. We suggest that perceptual information, when included with a source representation that is otherwise identical across participants, can directly improve analogical reasoning. Thus, we extend Pedone et al.'s results in two ways. First, we use kinesthetic information rather than visual information to affect analogical reasoning. Second, we show that when the likelihood of kinesthetic information being part of a source representation is influenced, analogical reasoning is affected.

\section{Experiment Overview}

Following a method originally developed by Gick and Holyoak $(1980,1983)$ and subsequently used by a number of researchers studying analogical problem solving (e.g., Beveridge \& Parkins, 1987; Catrambone \& Holyoak, 1989; Holyoak \& Koh, 1987; Keane, 1985; Pedone et al., 2001; Spencer \& Weisberg, 1986), we asked participants 
to try to solve Duncker's (1945) radiation problem after studying a story meant to serve as an analogical source. By comparing the rates at which participants solved the radiation problem when given the convergent force story versus the conventional story, we aimed to gauge the relative impact of the convergent force schema on the access and application of the analogy.

The source story used was a slightly modified version of the General story used by Gick and Holyoak (1983). The modification was for the purpose of another study, and pilot work found no difference in solution rates to the radiation target problem between the versions. In the source story, the men do not literally apply pressure on their target; the forces of the men instead combine like a generic resource prior to the resource's being used (e.g., similar to the way one might imagine fuel or money being combined). As a result, someone reading the General story might construct a perceptual representation of the events therein, but not necessarily one that included a convergent force schema.

Physical activity might directly structure one's representation of a source. Although we know of no studies that have tested this notion, studies have shown that physical activity can influence conceptual processing covertly. Klatzky, Pellegrino, McCloskey, and Doherty (1989), for example, found that when they asked participants to make apparently random hand gestures (e.g., pinching fingers into a point, balling fingers into a fist), the speed at which the participants were able to judge subsequent sentences as sensible or not (e.g., sentences such as "Aim a dart" or "Close a nail") depended significantly on whether the prior enactment was consistent with the sentence, even though the participants were blind to the meaning of the gestures.

If physical activity can influence representations, it should be possible to lead people to be more likely to include a convergent force schema in the representation of the General story by, for example, having people demonstrate the events in the story with their hands. If a convergent force schema is activated, it should, in turn, promote the analogy between the General story and the radiation problem.

To test this possibility, we designed an experiment with three conditions. In each case, participants were first asked to study the General story. Participants in the first condition - the verbal condition-were then asked to verbally recreate the story from memory. Participants in the second condition - the sketch condition-were asked to verbally recreate the story while simultaneously drawing an abstract diagram showing what had happened. Finally, participants in the third condition - the enactment condition - were asked to verbally recreate the story while simultaneously manipulating wood blocks to simulate what had happened. All participants were then given the radiation problem to solve, first without being explicitly told that the story and the problem were related, then with a hint to use the story.

Both sketching and enacting can enhance what people remember by providing additional retrieval cues. The ac- tivities associated with both enacting and sketching also might result in smoother and more productive recall, particularly as compared with conditions in which one's hands are restricted (Rauscher, Krauss, \& Chen, 1996). However, whereas both enacting and sketching might have certain advantages over physically restricted behavior, only enacting (manipulating blocks) offers the opportunity to experience convergent forces firsthand. Thus, participants in the enactment condition would be the most likely to directly activate a convergent force schema in association with events in the story.

In addition, other research suggests that enactment taps into representations beyond those that are purely verbal. A series of studies by Goldin-Meadow and her colleagues (Alibali, Bassok, Solomon, Syc, \& Goldin-Meadow, 1999; Garber \& Goldin-Meadow, 2002; Goldin-Meadow, Nusbaum, Kelly, \& Wagner, 2001) has indicated that gestures might reduce cognitive load and, most importantly for the present study, might illuminate people's problem representations in a way that goes beyond what is captured by purely verbal representations. Goldin-Meadow and colleagues found that verbal and gestural data sometimes diverge, particularly at choice points in a solution procedure. In one study, participants described problems both verbally and with gestures, and participants' solution strategies were then coded (Alibali et al., 1999). Of particular interest were the cases in which the verbal and gestural descriptions did not agree; in those cases, participants were relatively likely to choose a solution procedure consistent with their gestures. With respect to the present study, these results suggest not only that kinesthetic information can be a key element in problem and story representations, but that there might be genuine performance advantages when such information is present. We refer to what our participants did as enactment rather than gesture, in keeping with the terminology used in the literature. Gestures are considered to be spontaneous movements that typically accompany speech, whereas enactment is a set of intentional movements through which someone recreates a situation.

On the basis of the arguments and research outlined above, we predicted that participants in the enactment condition would perform better on the target problem than would those in either the verbal or the sketch condition, even though all the groups read the same source story and all the groups did fairly extensive processing of the source story.

\section{METHOD}

\section{Participants}

One hundred four participants were recruited from undergraduate psychology classes at Georgia Tech. Of the 104 participants, 39 were assigned to the enactment condition, 42 to the sketch condition, and 23 to the verbal condition. All received class credit for participating.

\section{Materials and Procedure}

The participants were told in advance that they would be participating in two separate studies: one concerning how people recalled stories in different modalities, the other concerning how people 
solved problems. All the participants were first given the General story (Appendix A) printed on a single sheet of paper and were asked to study it for $3 \mathrm{~min}$ so that they could accurately recreate it from memory later in the experiment. They were encouraged to repeatedly reread the story until they were told to stop; also, they were reminded that the purpose of the experiment was to study recall in different modalities. After they had read the story, they were asked to verbally recreate it in their own words, taking as much time as they needed.

The participants in the verbal condition were specifically told to recreate the story without using their hands if possible. The participants in the sketch condition were given a sheet of a paper $(8.5 \times$ 11 in.) and a black felt-tip pen, and were asked to draw a simple map-like diagram showing what had happened in the story while they verbally recreated it. They were instructed to draw simple shapes and contours to represent locations, and lines and arrows to represent movement and direction. They were discouraged from drawing anything in detail or labeling what they drew. The participants in the enactment condition were given three wood blocks and were told to slide them around on the desktop to show what had happened in the story while they verbally recreated it. The participants were told that one block, measuring $1.5 \times 1.5 \times 1.5$ in., was the dictator's bunker, while the other two blocks, measuring $1 \times 1.5 \times$ 3.5 in. each, were the armies. The blocks were initially aligned on the desk as in Figure 1.

The participants in all three conditions were asked to try to verbalize as much detail as they could. They were asked to concentrate in particular on what the protagonist did in the story - on both what he initially planned to do, and what he did in the end. Each participant's behavior was recorded on videotape.

After the verbal recall, the participants were told that the memory study was complete and that the second half of the session would be devoted to problem solving. They were given the radiation problem (Appendix B) and were asked to write down as many solutions as they could in $8 \mathrm{~min}$, focusing on those that were most plausible (Phase 1). They were advised that the problem was difficult but not impossible, and that no tricks were involved in its wording. After $8 \mathrm{~min}$, they were given a new sheet with the same problem printed on it and were told that they had $2.5 \mathrm{~min}$ to write a solution on the basis of the story that they had read earlier (Phase 2). After completing the radiation problem, they were given a short questionnaire asking them whether they had been familiar with the convergence solution to the radiation problem prior to the experiment and how easy and natural the recall task had been.

\section{RESULTS}

Solutions to the radiation problem in Phases 1, 2, and 3 were coded for whether they specified (1) the use of multiple beams of radiation, (2) the use of weak radiation (or "split" radiation), (3) the use of strong radiation,

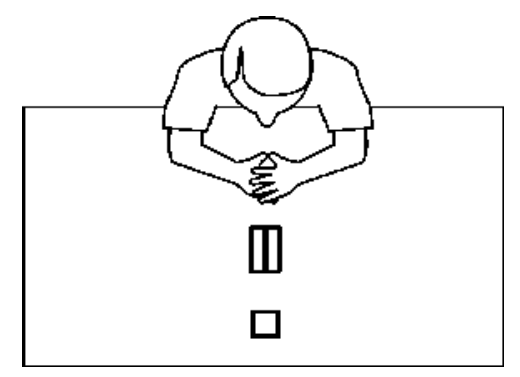

Figure 1. Initial configuration of blocks used during verbal recall in the enactment condition.
(4) the use of mixed strong and weak radiation, (5) the use of multiple treatments or doses of radiation over a period of time, and (6) the use of radiation from different angles, sides, or areas. Solutions that included Features 2 and either 1 or 6 , but not 3,4 , or 5, were considered convergence solutions - that is, solutions analogous to the solution in the General story.

Of the 104 participants, 17 indicated that they were already familiar with the convergence solution and were eliminated from further analysis. For the remaining 87 participants, solutions to the radiation problem in Phases 1 and 2 were coded following the scheme given above.

\section{Analogy Use as a Function of Condition}

In Phase 1 (before hint), 17 of 33 (52\%) participants in the enactment condition produced the convergence solution, as compared with 9 of 33 (27\%) in the sketch condition and 4 of $21(19 \%)$ in the verbal condition; this was a significant difference $\left[\chi^{2}(2, N=87)=7.21, p<.05\right]$. Pairwise comparisons showed that the enactment condition outperformed both the sketch $\left[\chi^{2}(1, N=66)=4.06\right.$, $p<.05]$ and the verbal $\left[\chi^{2}(1, N=54)=5.69, p<.05\right]$ conditions. The sketch and the verbal conditions did not differ significantly $\left[\chi^{2}(1, N=54)=0.48, p=.49\right]$.

Combining the solutions generated in Phases 1 and 2 reveals that $91 \%$ of the participants in the enactment condition produced the convergence solution, as compared with $85 \%$ in the sketch condition and $86 \%$ in the verbal condition. The differences were not significant $\left[\chi^{2}(2, N=\right.$ $87)=0.62, p=.73]$. The results are summarized in Table 1.

A number of additional analyses were undertaken to determine whether enactment and sketching influenced what people attended to and whether there were recall, enactment, or sketching features that were more strongly associated than were other features with the production of analogous solutions to the radiation problem.

\section{Recall as a Function of Condition}

Each participant's recall was reviewed on videotape and coded for the presence of 11 features causally related to the structure of the story. The frequency with which each feature appeared in the recall was then compared across all three conditions; no significant differences were found. The features, and the percentages of participants in each condition whose recall included them, are shown in Table 2.

The average number of causal features present was 5.8 for the enactment condition, 6.1 for the sketch condition, and 5.4 for the verbal condition. The differences among conditions were not significant $\left[F(2,84)=1.79, M S_{\mathrm{e}}=\right.$ $1.97, p=.17]$.

We compared the percentages of participants recalling the specific positive elements that constituted the convergence solution for the target problem. In Table 2, Elements 7,8 , and 9 correspond to the elements of the solution to the target problem that were considered key. A visual examination of the percentages for these elements in Table 2 shows no advantage for the enactment condition. Sketch- 
Table 1

Percentage of Participants Producing Convergence Solution to the Radiation Problem as a Function of Condition

\begin{tabular}{lccc}
\hline Condition & Before Hint & Total & $N$ \\
\hline Enactment & 52 & 91 & 33 \\
Sketch & 27 & 85 & 33 \\
Verbal & 19 & 86 & 21 \\
\hline
\end{tabular}

ing and enacting did not seem to change the likelihood of recalling these key elements.

\section{Analogy Use as a Function of Elements in Recall}

The number of features included in recall did not predict success on the radiation problem. If recall performance was divided into those who recalled at least 7 of the 10 elements $(n=59)$ versus those who recalled fewer than 7 $(n=28)$, the difference in the frequency of producing the convergence solution before the hint was not significant. In the former group, $39 \%$ produced the solution, whereas in the later group, $25 \%$ did so $\left[\chi^{2}(1, N=87)=1.69, p=\right.$ $.19]$. There was no difference after the hint either $[90 \%$ vs. $\left.82 \% ; \chi^{2}(1, N=87)=0.97, p=.32\right]$. We also tried cutoffs of 5 and 6 elements and found no group differences.

If analogy performance was assessed in terms of the quality of recall, no effect was observed. Specifically, participants who recalled Elements 7, 8, and 9 from Table 2 were labeled as higher recall quality participants, and those who did not recall all three elements were labeled as lower recall quality participants. There was no effect of higher $(n=26)$ or lower $(n=61)$ recall quality on before-hint performance [38\% vs. $33 \% ; \chi^{2}(1, N=87)=$ $0.26, p=.62]$ or after-hint performance [ $92 \%$ vs. $85 \%$; $\left.\chi^{2}(1, N=87)=0.89, p=.34\right]$. If the criteria for higher quality were raised so that Element 10 also had to be included, there was still no difference in before-hint performance $\left[40 \%\right.$ vs. $\left.34 \% ; \chi^{2}(1, N=87)=0.15, p=.70\right]$ or after-hint performance $\left[90 \%\right.$ vs. $87 \% ; \chi^{2}(1, N=87)=$ $0.071, p=.79]$.

\section{Analogy Use as a Function of Enactment}

The physical behavior of participants in the enactment condition was reviewed on videotape. Participants who actually made the blocks collide $(n=20)$ when demon- strating the soldiers converging on the bunker produced the convergence solution to the radiation problem more often than did those who did not make the blocks collide ( $n=13 ; 60 \%$ vs. $38 \%$, respectively). Thus the "collide" participants were $50 \%$ more likely prior to the hint to produce the convergence solution than were the "no-collide" participants. The difference in performance between the two groups was not significant $\left[\chi^{2}(1, N=33)=1.46\right.$, $p=.23]$, but this is partly a function of the low power of the test to detect a difference due to the small number of participants included in the analysis (which was necessarily restricted to those in the enactment condition). The solution rates after the hint $(95 \%$ vs. $85 \%)$ were not significantly different.

\section{Analogy Use as a Function of Sketches}

The drawings produced by participants in the sketch condition were coded for whether or not they included arrows depicting the initial head-on attack described in the General story and for whether or not they included arrows or lines depicting the final converging attack. If a sketch contained both features, it was labeled good, otherwise it was labeled poor. Of the 13 (of 33) participants in the sketch condition who produced good sketches, $31 \%$ produced the convergence solution to the radiation problem, as compared with $25 \%$ of those who produced poor sketches. This difference was not significant $\left[\chi^{2}(1, N=\right.$ $33)=0.13, p=.72]$. Production of convergence solutions after the hint (including those produced before the hint) also did not differ $\left[92 \%\right.$ vs. $80 \% ; \chi^{2}(1, N=33)=$ $0.93, p=.34]$.

\section{DISCUSSION}

Our goal in the present work was to examine whether a story's perceptual context, independent of its predicate structure, would influence whether and how people could use the story to construct an analogy. Specifically, we wished to examine whether kinesthetic information would affect access of a source story when one worked on a target problem and whether this information also would affect the application of the analogy once noticed. We found that when participants performed physical activities hypothesized to make the activation of the kinesthetic

Table 2

Percentage of Participants in Each Condition

Recalling Particular Source Story Features

\begin{tabular}{lccccccccccr}
\hline & \multicolumn{10}{c}{ Feature } \\
\cline { 2 - 12 } Condition & 1 & 2 & 3 & 4 & 5 & 6 & 7 & 8 & 9 & 10 & 11 \\
\hline Enactment & 81 & 33 & 74 & 33 & 81 & 33 & 89 & 41 & 78 & 44 & 7 \\
Sketch & 84 & 41 & 84 & 15 & 90 & 25 & 100 & 30 & 85 & 35 & 20 \\
Verbal & 75 & 18 & 83 & 20 & 75 & 18 & 98 & 40 & 83 & 48 & 5 \\
\hline
\end{tabular}

Note-Features are numbered as follows. (1) The bunker or compound was centrally located;

(2) the bunker was strong or well armed; (3) the rebel army was large and/or strong; (4) the rebel army would have to attack together and/or with full force; (5) mines detected large, heavy, and/or powerful things; (6) the rebel army was too large; (7) the rebel army split, or two smaller rebel groups were used; (8) the groups were small enough to clear the mines; (9) the groups moved to or attacked from different directions or areas; (10) the groups converged or met at the bunker; (11) the groups attacked with full force. 
information likely during story processing, access (but not application) improved. The sketching task did not aid performance. On the basis of the assumption that participants in the enactment condition would be more likely to include kinesthetic information in the story representation, the results provide support for the hypothesis that the conceptual structure used in accessing source examples can be perceptually represented.

Catrambone (2002) found an effect of structural features on the access of analogues, and he argued that this finding was due partly to a more sensitive measure of access (reading time) than those used in prior studies. In the present experiment, we also found a benefit in access due to structural features, but rather than its being due to a particularly sensitive measure, we suggest that it is due to the increased likelihood of the presence of relevant kinesthetic information in the source story representation for the enactment condition.

We carefully reviewed the content of each participant's verbal recall and found no significant differences across conditions. This result suggests that the verbal recall might not have been as important for solution performance as were the physical behaviors accompanying it. This is consistent with the findings of Goldin-Meadow and her colleagues (Alibali et al., 1999; Goldin-Meadow et al., 2001) suggesting that gestures might tap into a separate representation and provide processing advantages beyond the verbal representation.

The advantage conferred by enacting over both sketching and purely verbal recall does not seem to be due to any effect that enacting might have had on what and how much was verbally recalled. This is congruent with the claim made earlier-namely, that enacting while reading the General story would make a person more likely to activate a convergent force schema when processing the story, but would not necessarily make the story inherently more memorable. A kinesthetic schema might encode structure, but it is the fact that one can directly "read off" salient kinesthetic attributes (e.g., strong at the center, weak at the periphery) that makes it useful. Furthermore, because differences in the way the story was recalled (verbal vs. verbal+sketch vs. verbal+enactment) likely would not have led to differences in the activation of superficial features (at least not those linked in any obvious way to the target problem), we conclude that the type of recall affected the perceptual representation of conceptual structure.

In the present study, participants in the enactment condition were required to interact with the blocks. A future study could use spontaneous gestures during verbal retelling as another index of perceptual structure. The prediction is that participants who spontaneously produce gestures evoking converging forces-versus a set of individual linear forces - would be more likely to succeed on the transfer problem than would participants who do not. Such a result would help tease apart whether the crucial feature is convergence of forces or a collection of linear forces.

Another extension to this work would be to examine whether there are systematic differences in the effects of (spontaneous) gestures and (guided) enactments on subse- quent analogical problem solving. Can enactment reliably lead to improvements in problem solving by people who normally do not produce gestures when studying materials? In addition, can certain gestures and enactments be misleading? If so, are the effects of gestures and enactments about the same?

Although our findings are consistent with the claim that conceptual structure can be perceptually represented, they do not rule out the possibility that other types of representations, such as amodal representations and text-level representations, could be used in other circumstances. Our results could be read as showing that even if amodal representations are used to help form an analogy between a source and a target, the addition of perceptual information can make a source more accessible. Perceptual information might often be a component of conceptual structure. The contribution of perceptual information to the access and application of analogies should be considered systematically in future work, particularly when researchers propose representational schemes.

\section{REFERENCES}

Alibali, M. W., Bassok, M., Solomon, K. O., Syc, S. E., \& GoldinMEadow, S. (1999). Illuminating mental representations through speech and gesture. Psychological Science, 10, 327-333.

Anderson, R. C. (1984). Some reflections on the acquisition of knowledge. Educational Researcher, 13, 5-10.

Barsalou, L. W. (1999). Perceptual symbol systems. Behavioral \& Brain Sciences, 22, 577-609.

Beveridge, M., \& PARKINS, E. (1987). Visual representation in analogical problem solving. Memory \& Cognition, 15, 230-237.

Catrambone, R. (2002). The effects of surface and structural feature matches on the access of story analogs. Journal of Experimental Psychology: Learning, Memory, \& Cognition, 28, 318-334.

Catrambone, R., \& Holyoak, K. J. (1989). Overcoming contextual limitations on problem-solving transfer. Journal of Experimental Psychology: Learning, Memory, \& Cognition, 15, 1147-1156.

Duncker, K. (1945). On problem-solving. Psychological Monographs, 58(5, Whole No. 9).

FINCHER-KIEFER, R. (2001). Perceptual components of situation models. Memory \& Cognition, 29, 336-343.

Garber, P., \& Goldin-Meadow, S. (2002). Gesture offers insight into problem-solving in adults and children. Cognitive Science, 26, 817-831.

Gentner, D., Rattermann, M. J., \& Forbus, K. D. (1993). The roles of similarity in transfer: Separating retrievability from inferential soundness. Cognitive Psychology, 25, 524-575.

Gick, M. L., \& Holyonk, K. J. (1980). Analogical problem solving. Cognitive Psychology, 12, 306-335.

Gick, M. L., \& HolyOAK, K. J. (1983). Schema induction and analogical transfer. Cognitive Psychology, 15, 1-38.

GlenberG, A. M. (1997). What memory is for. Behavioral \& Brain Sciences, 20, 1-19.

Goldin-Meadow, S., Nusbaum, H., Kelly, S. D., \& Wagner, S. (2001). Explaining math: Gesturing lightens the load. Psychological Science, 12, 516-522.

HolyoAK, K. J., \& KoH, K. (1987). Surface and structural similarity in analogical transfer. Memory \& Cognition, 15, 332-340.

JoHnson, M. (1987). The body in the mind: The bodily basis of meaning, imagination, and reason. Chicago: University of Chicago Press.

KEANE, M. [T.] (1985). On drawing analogies when solving problems: A theory and test of solution generation in an analogical problemsolving task. British Journal of Psychology, 76, 449-458.

Keane, M. T., Ledgeway, T., \& Duff, S. R. S. (1994). Constraints on analogical mapping: A comparison of three models. Cognitive Science, 18, 387-438.

Klatzky, R. L., Pellegrino, J. W., McCloskey, B. P., \& Doherty, S. 
(1989). Can you squeeze a tomato? The role of motor representations in semantic sensibility judgments. Journal of Memory \& Language, 28, 56-77.

LAKOFF, G. (1987). Women, fire, and dangerous things: What categories reveal about the mind. Chicago: University of Chicago Press.

MANDLER, J. M. (1992). How to build a baby: II. Conceptual primitives. Psychological Review, 99, 587-604.

Pecher, D., Zeelenberg, R., \& Barsalou, L. W. (2003). Verifying properties from different modalities for concepts produces switching costs. Psychological Science, 14, 119-124.

Pedone, R., Hummel, J. E., \& Holyoak, K. J. (2001). The use of diagrams in analogical problem solving. Memory \& Cognition, 29, 214221.

Rauscher, F. H., Krauss, R. M., \& Chen, Y. (1996). Gesture, speech, and lexical access: The role of lexical movements in speech production. Psychological Science, 7, 226-231.

Richardson, D. C., Spivey, M. J., Edelman, S., \& Naples, A. (2001).
Language is spatial: Experimental evidence for image schemas of concrete and abstract verbs. In J. D. Moore \& K. Stenning (Eds.), Proceedings of the 23rd Annual Meeting of the Cognitive Science Society (pp. 873-878). Mahwah, NJ: Erlbaum.

Solomon, K. O., \& Barsalou, L. W. (2001). Representing properties locally. Cognitive Psychology, 43, 129-169.

SPENCER, R., \& WeISBERG, R. (1986). Context-dependent effects on analogical transfer. Memory \& Cognition, 14, 442-449.

STANFIELD, R. A., \& ZWAan, R. A. (2001). The effect of implied orientation derived from verbal context on picture recognition. Psychological Science, 12, 153-156.

Wharton, C. M., Holyoak, K. J., Downing, P. E., Lange, T. E., Wickens, T. D., \& Melz, E. R. (1994). Below the surface: Analogical similarity and retrieval competition in reminding. Cognitive Psychology, 26, 64-101.

WolfF, P., \& Song, G. (2003). Models of causation and the semantics of causal verbs. Cognitive Psychology, 47, 276-332.

\section{APPENDIX A}

General Story

In the woods in the middle of a small country is a dictator's compound. Although the dictator and his men are usually quiet, they pose a potential danger to the people in the surrounding area and therefore must be constantly monitored. At any time they could become agitated and start causing trouble.

One day, John, a rebel leader, decides to do something about the dictator and approaches the compound with a big army of men. He knows that the dictator lives in a bunker in the center of the compound, and he is pretty sure that if he has his entire army attack that spot he can take out the dictator and force the rest of the dictator's men to disband. He also knows, though, that the compound is filled with mines and that the mines will probably explode in a blizzard of shrapnel if he sends his army through the compound all at once, simply because of the size of his army and the sensitivity of the mines.

After thinking about his dilemma, John decides to split his big army into two smaller groups. He positions the two smaller groups at two different points around the compound and has them march in toward the center from different directions, using a two-way radio to communicate with both of them at once. Because the two groups are relatively small, they pass safely through the compound without detonating the mines, but as they meet in the center their combined force is enough to take out the dictator. Once the dictator is dead, the rest of the dictator's men leave without a fight.

\section{APPENDIX B \\ The Radiation Problem}

Suppose you are a doctor faced with a patient who has a life-threatening stomach tumor. It is impossible to operate on the patient, but unless the tumor is destroyed the patient will die. A new form of radiation may be used to destroy the tumor, but there is a complication: A beam of radiation strong enough to destroy the tumor will invariably destroy any healthy tissue it passes through as well, thus killing the patient in the process. A weaker beam of radiation, on the other hand, won't harm the healthy tissue it passes through, but it won't destroy the tumor either, no matter how long it is used.

What type of procedure might be used to destroy the tumor using the new form of radiation, without destroying any healthy tissue and without cutting the patient open to expose the tumor?

(Manuscript received January 14, 2004;

revision accepted for publication May 19, 2005.) 